\title{
REALIZATION OF THE LEVEL ONE STANDARD $\widetilde{\mathbf{C}}_{2 k+1}$-MODULES
}

\author{
KAILASH C. MISRA
}

\begin{abstract}
In this paper we study the level one standard (or irreducible integrable highest weight) modules for the affine symplectic Lie algebras. In particular, we give concrete realizations of all level one standard modules for the affine symplectic Lie algebras of even rank.
\end{abstract}

\section{INTRODUCTION}

In recent years explicit realizations of nontrivial representations of affine Lie algebras have attracted the attention of many researchers because of their surprising connections with different areas of mathematics and physics. Among these realizations the 'principal realizations' and the 'untwisted (homogeneous) realizations' have proved to be especially useful. In 1978 Lepowsky and Wilson [15] constructed the basic modules-level one standard modules (i.e. irreducible integrable highest weight modules)-for $A_{1}^{(1)}$ in the principal realization. In [10] this construction was generalized to the level one standard modules for all simply-laced affine Lie algebras. Frenkel and Kac [5] and Segal [26] have given explicit construction of these modules in the untwisted realization. The constructions of these modules in the general realization are given in $[9,12]$. Since then some higher level standard modules for certain simply-laced affine Lie algebras have also been constructed (e.g. $[14,17,18,19,21,22,25])$. However, less is known regarding explicit constructions of standard modules for nonsimply-laced affine Lie algebras-that is, affine Lie algebras of type ' $B$ ', ' $C$ ', ' $F$ ' and ' $G$ '. In the last section of [10] Kac, Kazhdan, Lepowsky and Wilson constructed reducible level one modules for the non-simply-laced affine Lie algebras in the principal realization by taking fixed points of Dynkin-diagraminduced automorphisms. Recently, in the untwisted realization the analogous representations (again reducible) have been constructed in [ 3 and 6].The level one standard modules for the affine orthogonal Lie algebras $B_{n}^{(1)}$ have been explicitly realized in $[4,8,13$ and 20] from different view points. In [20] Mandia has constructed the level one standard modules for the affine Lie algebras $F_{4}^{(1)}$ and $G_{2}^{(1)}$. In [23] the author gave explicit constructions of the level one standard modules for the affine symplectic Lie algebras of rank 3 and 4.

Received by the editors July 13, 1989. Presented to the 847 th meeting of the AMS, Pheonix, Arizona, January 14, 1989.

1980 Mathematics Subject Classification (1985 Revision). Primary 17B65. 
In this paper we give explicit realization of the level one standard modules for all affine symplectic Lie algebras of even rank in the principal realization. Following the patterns of many of the papers mentioned above, we first start from the fact that the structure of a standard module $L$ is determined by the structure of the vacuum space $\Omega(L)$ for the principal Heisenberg subalgebra $\underline{s}$ in $L$ (see $\S 1$ ). To study $\Omega(L)$ we make use of the $Z$-operators introduced by Lepowsky and Wilson $[16,18]$. We recall necessary facts about these operators in $\S 1$. In $\S 2$ we prove several generating function identities for these operators. In $\S 3$ we use these identities to determine (Theorem 3.15) a suitable spanning set for $\Omega(L)$. We then show (Theorem 3.16) using the generalized RogersRamanujan identities, that our spanning set is in fact a basis for $\Omega(L)$, when $L$ is a level one standard module for any affine symplectic Lie algebra of even rank.

\section{Preliminaries}

In this section we will recall some notations and facts from [23]. There will be minor changes in the notations which will be self-explanatory. For more details see [23].

Consider the (complex) simple Lie algebra $s \underline{s}(2 n, \mathbb{C}), n \geq 2$. The associated affine Lie algebra $\underline{s l}(2 n, \mathbb{C})^{\wedge}$ has a basis $($ see $[10,25])$,

$$
\{c, B(j), \underline{X}(m, i) \mid i, j \in \mathbb{Z}, j \not \equiv 0 \bmod (2 n), m=1, \ldots, 2 n-1\},
$$

where $c$ is a central element (suitably normalized), $B(j)=E^{j} \otimes t^{j}$ and $\underline{X}(m, i)$ $=D^{m} E^{i} \otimes t^{i}$. Here $t$ is an indeterminate, $D=\operatorname{diag}\left(\omega, \ldots, \omega^{2 n-1}, 1\right)$, where $\omega$ is a primitive $(2 n)$ th root of unit and

$$
E=\left(\begin{array}{ccccc}
0 & 1 & 0 & \cdots & 0 \\
0 & 0 & 1 & \cdots & 0 \\
& \cdots & & \cdots & \\
0 & 0 & 0 & \cdots & 1 \\
1 & 0 & 0 & \cdots & 0
\end{array}\right) \in \underline{s l}(2 n, \mathbb{C}) .
$$

Consider the order two automorphism

$$
\theta: x \mapsto-y x^{t} y^{-1}
$$

of $\underline{s l}(2 n, \mathbb{C})$, where $y=\left((-1)^{i+1} \delta_{i, 2 n-j+1}\right)_{i, j=1}^{2 n}$ and $x^{t}$ denotes the transpose of $x$. Observe that $\theta$ is induced by the usual order two graph automorphism of the Dynkin diagram of $\underline{s l}(2 n, \mathbb{C})$. Extend $\theta$ to an automorphism of $\underline{s l}(2 n, \mathbb{C})^{\wedge}$, again denoted by $\theta$, by defining

$$
\left\{\begin{array}{l}
\theta\left(x \otimes t^{i}\right)=\theta(x) \otimes t^{i}, \quad \text { for all } x \in \underline{s l}(2 n, \mathbb{C}), \text { and } \\
\theta(c)=c .
\end{array}\right.
$$

Observe that

$$
\theta(B(j))= \begin{cases}B(j), & \text { for all } j \text { odd }, \\ -B(j), & \text { for all even } j \not \equiv 0(\bmod 2 n),\end{cases}
$$


and

$$
\theta(\underline{X}(m, i))=(-1)^{i+1} \omega^{m(1-i)} \underline{X}(2 n-m, i)
$$

for all $i \in \mathbb{Z}$ and $m=1,2, \ldots, 2 n-1$. Then it is known that (see [23, Proposition 2.1]) the set

$$
\{c, B(j), X(m, i) \mid i, j \in \mathbb{Z}, j \text { odd }, m=1,2, \ldots, n\}
$$

where

$$
X(m, i)=\frac{1}{2}\{\underline{X}(m, i)+\theta \underline{X}(m, i)\}
$$

forms a basis of the affine symplectic Lie algebra $C_{n}^{(1)}=\underline{s p}(2 n, \mathbb{C})^{\wedge}$. Denote

$$
\underline{s}^{ \pm}=\coprod_{\substack{j>0 \\ j \text { odd }}} \mathbb{C} B( \pm j) \text { and } \underline{s}=\underline{s}^{-} \oplus \mathbb{C} c \oplus \underline{s}^{+} .
$$

Then $\underline{s}$ is a Heisenberg subalgebra of $\underline{s p}(2 n, \mathbb{C})^{\wedge}$, called the principal Heisenberg subalgebra.

Let $\left\{E_{i}, H_{i}, F_{i} \mid 0 \leq i \leq 2 n-1\right\}$ be the canonical set of generators of the affine Lie algebra $\underline{s l}(2 n, \mathbb{C})^{\wedge}$. Observe that $c=\sum_{i=0}^{2 n-1} H_{i}$. Set

$$
\begin{array}{lll}
e_{0}=E_{0}, & h_{0}=H_{0}, & f_{0}=F_{0}, \\
e_{n}=E_{n}, & h_{n}=H_{n}, & f_{n}=F_{n}, \\
e_{i}=E_{i}+E_{2 n-i}, & h_{i}=H_{i}+H_{2 n-i}, & f_{i}=F_{i}+F_{2 n-i},
\end{array}
$$

for $i=1,2, \ldots, n-1$. Then $\left\{e_{i}, h_{i}, f_{i} \mid 0 \leq i \leq n\right\}$ forms a set of canonical generators $($ see $[10,23])$ for the affine symplectic Lie algebra $C_{n}^{(1)}=$ $\underline{s p}(2 n, \mathbb{C})^{\wedge}$.

Let $d$ be the derivation of $\underline{s l}(2 n, \mathbb{C})^{\wedge}$ given by

$$
d\left(E_{i}\right)=E_{i}, \quad d\left(F_{i}\right)=-F_{i} \text { and } d\left(H_{i}\right)=0,
$$

for $i=0,1, \ldots, 2 n-1$. Observe that

$$
\begin{gathered}
d(B(j))=j B(j), \quad d(X(m, i))=i X(m, i), \\
d(\underline{X}(m, i))=i \underline{X}(m, i) \text { and } d(c)=0 .
\end{gathered}
$$

Note that $d$ restricted to $\underline{s p}(2 n, \mathbb{C})^{-}$, again denoted by $d$, is a derivation of $\underline{s p}(2 n, \mathbb{C})^{\wedge}$ with

$$
d\left(e_{i}\right)=e_{i}, \quad d\left(f_{i}\right)=-f_{i} \text { and } d\left(h_{i}\right)=0,
$$

for $i=0,1, \ldots, n$. Form the semidirect product Lie algebras

$$
\underline{s l}(2 n, \mathbb{C})^{\sim}=\underline{s l}(2 n, \mathbb{C})^{\wedge} \oplus \mathbb{C} d
$$

and

$$
\tilde{\mathfrak{g}}=\underline{s p}(2 n, \mathbb{C})^{\sim}=\underline{s p}(2 n, \mathbb{C})^{\wedge} \oplus \mathbb{C} d
$$

Denote

$$
\tilde{\mathfrak{g}}_{i}=\{x \in \tilde{\mathfrak{g}} \mid[d, x]=i x\}, \quad i \in \mathbb{Z} .
$$


Then

$$
\tilde{\mathfrak{g}}=\coprod_{i \in \mathbb{Z}} \tilde{\mathfrak{g}}_{i}
$$

which gives a $\mathbb{Z}$-gradation (called the principal gradation) for $\tilde{\mathfrak{g}}$, in the sense that $\left[\tilde{\mathfrak{g}}_{i}, \tilde{\mathfrak{g}}_{j}\right] \subseteq \tilde{\mathfrak{g}}_{i+j}$. This induces naturally a $\mathbb{Z}$-gradation in the universal enveloping algebra $\mathscr{U}(\tilde{\mathfrak{g}})$,

$$
\mathscr{U}(\tilde{\mathfrak{g}})=\coprod_{i \in \mathbb{Z}} \mathscr{U}(\tilde{\mathfrak{g}})_{i} .
$$

For $\mathfrak{h}=\operatorname{span}\left\{h_{1}, h_{2}, \ldots, h_{n}\right\}$, set

$$
\tilde{\mathfrak{h}}=\mathfrak{h} \oplus \mathbb{C} c \oplus \mathbb{C} d \subseteq \tilde{\mathfrak{g}} .
$$

Then $\tilde{\mathfrak{h}}$ is an abelian subalgebra of $\tilde{\mathfrak{g}}$ which is spanned by $\left\{h_{0}, h_{1}, \ldots, h_{n}, d\right\}$.

Let $\lambda \in \tilde{\mathfrak{h}}^{*}$. A $\tilde{\mathfrak{g}}$-module $V$ generated by a vector $v_{\lambda} \neq 0$ such that $e_{i} \cdot v_{\lambda}=0$ for $i=0,1, \ldots, n$ and $h \cdot v_{\lambda}=\lambda(h) v_{\lambda}$ for all $h \in \tilde{\mathfrak{h}}$, is called a highest weight module with highest weight $\lambda$. Such a vector $v_{\lambda}$ is called a highest weight vector and is unique up to a scalar multiple. The scalar $\lambda(c)$ is said to be the level of $V$. A highest weight $\tilde{\mathfrak{g}}$-module with highest weight $\lambda$ and corresponding highest weight vector $v_{\lambda}$ is called a standard (or integrable highest weight) $\tilde{\mathfrak{g}}$-module if there is an integer $r \geq 1$ such that $f_{i}^{r} \cdot v_{\lambda}=0,0 \leq i \leq n$, which in turn implies that $\lambda$ is dominant integral, that is, $\lambda\left(h_{i}\right) \in \mathbb{N}$, for $0 \leq i \leq n$ (see [7, 11]). For each dominant integral $\lambda \in \tilde{\mathfrak{h}}^{*}$, there is a unique (up to isomorphism) standard $\tilde{\mathfrak{g}}$-module $L(\lambda)$ and it is irreducible (see $[7,11])$. For convenience we will restrict our attention to $L(\lambda)$ when $\lambda(d)=0$, so that $\lambda \in \operatorname{span}\left\{h_{i}^{*} \mid 0 \leq\right.$ $i \leq n\} \subseteq \tilde{\mathfrak{h}}^{*}$ where $h_{i}^{*}\left(h_{j}\right)=\delta_{i, j}$ and $h_{i}^{*}(d)=0,0 \leq i, j \leq n$.

A standard $C_{n}^{(1)}$-module by definition is the restriction to $C_{n}^{(1)}=\underline{s p}(2 n, \mathbb{C})^{-}$ of a standard $s \underline{p}(2 n, \mathbb{C})^{\sim}$-module. The standard modules for $\underline{s l}(\overline{2 n}, \mathbb{C})^{\wedge}$ or $\underline{s l}(2 n, \mathbb{C})^{\sim}$ are defined in an analogous way.

For the standard $\tilde{\mathfrak{g}}$-module $L=L(\lambda)$ (with $\lambda(d)=0$ ) denote by $L_{i} \subseteq L(\lambda)$ the eigenspace of $d$ with eigenvalue $i \in \mathbb{Z}$. Then

$$
L=L(\lambda)=\coprod_{i \leq 0} L_{i}
$$

with $L_{0}=\mathbb{C} v_{\lambda}, L_{i}=\mathscr{U}(\tilde{\mathfrak{g}})_{i} v_{\lambda}$ and $\operatorname{dim} L_{i}<\infty$. Note that for $i \leq 0, v \in L_{i}$, we have $d \cdot v=i v$. Hence we call (see [18]) the set of elements in $L_{i}$ to be the set of homogeneous elements of degree $i$. In particular, we say that $T \in \operatorname{End}(L(\lambda))$ is homogeneous of degree $z \in \mathbb{C}$ if $[d, T]=z T$. Observe that $T \in \operatorname{End}(L(\lambda))$ has degree $z \in \mathbb{C}$ if and only if $T L_{i} \subseteq L_{i+z}$ for all $i \in \mathbb{Z}$. Define the principal character $\chi(L(\lambda))$ of $L(\lambda)$ by

$$
\chi(L(\lambda))=\sum_{i \geq 0}\left(\operatorname{dim} L_{-i}\right) q^{i}
$$

where $q$ is an indeterminate. Then $\chi(L(\lambda))$ has a known product expansion (for example, see [22, Formula 1.1]). 
In this paper we will focus our attention to the level one standard (or integrable highest weight) $\tilde{\mathfrak{g}}$-modules $L(\lambda)$ with $\lambda(d)=0$. To be more precise, we will study the standard $\tilde{\mathfrak{g}}$-modules $L\left(h_{i}^{*}\right), i=0,1, \ldots, n$. Let us write $t=[n / 2]+1$, where [·] denotes the greatest integer. From here on $L(\lambda)$ will always denote a level one standard $\tilde{\mathfrak{g}}$-module with $\lambda(d)=0$. By direct computation (cf. [24]) it can be easily shown that for $i=0,1, \ldots, t-1$, and $i \neq n / 2$, we have

$$
\chi\left(L\left(h_{i}^{*}\right)\right)=\chi\left(L\left(h_{n-i}^{*}\right)\right)=F \prod_{\substack{k>0 \\ k \neq 0, \pm(i+1) \bmod (n+2)}}\left(1-q^{2 k}\right)^{-1}
$$

and for $i=n / 2$ (i.e. $n$ even), we have

$$
\chi\left(L\left(h_{i}^{*}\right)\right)=F \prod_{\substack{k>0 \\ k \neq 0,(i+1) \bmod (n+2)}}\left(1-q^{2 k}\right)^{-1} \cdot \prod_{\substack{k>0 \\ k \equiv(i+1) \bmod (n+2)}}\left(1-q^{2 k}\right),
$$

where

$$
F=\prod_{k>0}\left(1-q^{2 k-1}\right)^{-1}
$$

It is important to observe that $\chi\left(L\left(h_{i}^{*}\right)\right)$ differs from the product side of the generalized Rogers-Ramanujan identities (with ' $q$ ' replaced by ' $q$ ') due to Gordon, Andrews and Bressoud (see $[1,2]$ ) by a simple factor.

Recall the subalgebra $\underline{s}^{+}($see $(1.8))$. Let $\underline{p}$ denote the subalgebra $\underline{s}^{+} \oplus$ $\mathbb{C} c$. For the standard $\tilde{\mathfrak{g}}$-module $L=L(\lambda)$ denote by $\Omega(L)=\Omega(L(\lambda)$ ) (or by $\Omega$ if there is no confusion) its vacuum space with respect to the Heisenberg subalgebra $\underline{s}$,

$$
\Omega(L(\lambda))=\left\{v \in L(\lambda) \mid \underline{s}^{+} \cdot v=0\right\},
$$

which is graded. Then the map (cf. $[16,18]$ ),

$$
\begin{gathered}
f: U(\underline{s}) \otimes_{\mathscr{U}(\underline{p})} \Omega(L(\lambda)) \rightarrow L(\lambda) \\
u \otimes w \mapsto u \cdot w
\end{gathered}
$$

for all $u \in \mathscr{U}(\underline{s}), w \in \Omega(L(\lambda))$, is an $\underline{s}$-module isomorphism. In particular

$$
\chi(L(\lambda))=F \cdot \chi(\Omega(L(\lambda)))
$$

where

$$
F=\chi\left(\mathscr{U}\left(\underline{s}^{-}\right)\right)=\prod_{k>0}\left(1-q^{2 k-1}\right)^{-1} .
$$

Hence, in order to give concrete realizations of the level one standard $\tilde{\mathfrak{g}}$-modules $L\left(h_{i}^{*}\right), 0 \leq i \leq n$, it is enough to give explicit constructions of the corresponding vacuum spaces $\Omega\left(L\left(h_{i}^{*}\right)\right), 0 \leq i \leq n$. This is exactly what we will do in this paper. It follows from $(1.20),(1.21)$ and $(1.25)$ that for $i=0,1, \ldots, n$, $i \neq n / 2$. We have

$$
\chi\left(\Omega\left(L\left(h_{i}^{*}\right)\right)\right)=\chi\left(\Omega\left(L\left(h_{n-i}^{*}\right)\right)\right)=\prod_{\substack{k>0 \\ k \neq 0, \pm(i+1) \bmod (n+2)}}\left(1-q^{2 k}\right)^{-1}
$$


and for $i=n / 2$ (i.e. $n$ even), we have

$$
\text { (1.27) } \chi\left(\Omega\left(L\left(h_{i}^{*}\right)\right)\right)=\prod_{\substack{k>0 \\ k \neq 0,(i+1) \bmod (n+2)}}\left(1-q^{2 k}\right)^{-1} \cdot \prod_{\substack{k>0 \\ k \equiv(i+1) \bmod (n+2)}}\left(1-q^{2 k}\right) \text {. }
$$

For a formal indeterminate $\zeta$, denote by $\operatorname{End}(L(\lambda))\{\zeta\}$ the $\mathbb{C}$-vector space of formal Laurent series in $\zeta$ with coefficients in $\operatorname{End}(L(\lambda)) . \operatorname{In} \operatorname{End}(L(\lambda))\{\zeta\}$ define, for $k \in \mathbb{Z}$,

$$
\underline{E}^{ \pm}(k, \zeta)=\exp \left( \pm \sum_{\substack{j>0 \\ j \neq 0 \bmod (2 n)}}\left(\omega^{\mp k j}-1\right) B( \pm j) \zeta^{ \pm j} / j\right)
$$

$$
E^{ \pm}(k, \zeta)=\exp \left( \pm \sum_{\substack{j>0 \\ j \text { odd }}}\left(\omega^{\mp k j}-1\right) B( \pm j) \zeta^{ \pm j} / j\right)
$$

$$
\begin{aligned}
\underline{X}(m, \zeta) & =\sum_{i \in \mathbb{Z}} \underline{X}(m, i) \zeta^{i}, \quad m=1,2, \ldots, 2 n-1, \\
X(m, \zeta) & =\sum_{i \in \mathbb{Z}} X(m, i) \zeta^{i}, \quad m=1,2, \ldots, n, \\
\delta(\zeta) & =\sum_{i \in \mathbb{Z}} \zeta^{i} \text { and } D \delta(\zeta)=\sum_{i \in \mathbb{Z}} i \zeta^{i},
\end{aligned}
$$

where 'exp' means the formal exponential series. Observe that

$$
\underline{E}^{ \pm}(k, \zeta)=E^{ \pm}(k, \zeta) \exp \left( \pm P^{ \pm}(k, \zeta)\right)
$$

where

$$
P^{ \pm}(k, \zeta)=\sum_{\substack{j>0 \\ j \neq 0 \text { even } j \neq 0 \bmod (2 n)}}\left(\omega^{\mp k j}-1\right) B( \pm j) \zeta^{ \pm j} / j
$$

Now define the elements

$$
Z(m, \zeta)=E^{-}(m, \zeta) X(m, \zeta) E^{+}(m, \zeta)
$$

for $m=1,2, \ldots, n$, in $\operatorname{End}(L(\lambda))\{\zeta\}$ (with $\lambda(c)=1$ ). These elements are well-defined since the grading of $L(\lambda)$ is truncated from above. Let

$$
Z(m, \zeta)=\sum_{i \in \mathbb{Z}} Z(m, i) \zeta^{i}
$$

for $m=1,2, \ldots, n$, where $Z(m, i) \in \operatorname{End}(L(\lambda))$ is the homogeneous component of degree $i$ of $Z(m, \zeta)$. Denote by $\underline{Z}=\underline{Z}(L(\lambda))$ the subalgebra (see 
$[16,18])$ of $\operatorname{End}(L(\lambda))$ generated by

$$
\{Z(m, i) \mid i \in \mathbb{Z}, m=1,2, \ldots, n\} .
$$

Then (see $[16,18])$ the algebra $\underline{Z}$ centralizes the action of the Heisenberg subalgebra $\underline{s}$ on $L(\lambda)$. In particular, the algebra $\underline{Z}$ preserves the vacuum space $\Omega=\Omega(L(\lambda))$ of $L(\lambda)$ with respect to $\underline{s}$. Furthermore, (see $[16,18]$ ) we have

$$
\Omega=\underline{Z} \cdot v_{\lambda}=\operatorname{span}_{\mathbb{C}}\left\{Z\left(m_{1}, i_{1}\right) \cdots Z\left(m_{k}, i_{k}\right) \cdot v_{\lambda}\right\}
$$

where $i_{j} \in \mathbb{Z}$ and $1 \leq m_{j} \leq n$, for each $j$. For two commuting indeterminates $\zeta_{1}$ and $\zeta_{2}$, and $m, l=1,2, \ldots, n$, denote

$$
F\left(m, l ; \zeta_{1}, \zeta_{2}\right)=\left\{\frac{\left(1-\frac{\zeta_{1}}{\zeta_{2}}\right)\left(1+\frac{\omega^{l} \zeta_{1}}{\zeta_{2}}\right)\left(1+\frac{\zeta_{1}}{\omega^{m} \zeta_{2}}\right)\left(1-\frac{\omega^{l} \zeta_{1}}{\omega^{m} \zeta_{2}}\right)}{\left(1+\frac{\zeta_{1}}{\zeta_{2}}\right)\left(1-\frac{\omega^{l} \zeta_{1}}{\zeta_{2}}\right)\left(1-\frac{\zeta_{1}}{\omega^{m} \zeta_{2}}\right)\left(1+\frac{\omega^{l} \zeta_{1}}{\omega^{m} \zeta_{2}}\right)}\right\}^{\frac{1}{2}}
$$

Theorem 1.1 [23, Theorem 2.4]. Let $L(\lambda)$ be a level one standard $\tilde{\mathfrak{g}}$-module with $\lambda(d)=0$, and $\zeta_{1}, \zeta_{2}$ be two commuting indeterminates. Then on $L(\lambda)$, for $m, l=1,2, \ldots, n$, we have

$$
\begin{gathered}
F\left(m, l ; \zeta_{1}, \zeta_{2}\right) Z\left(m, \zeta_{1}\right) Z\left(l, \zeta_{2}\right)-F\left(l, m ; \zeta_{2}, \zeta_{1}\right) Z\left(l, \zeta_{2}\right) Z\left(m, \zeta_{1}\right) \\
=\left\{\begin{array}{c}
-\frac{1}{2} \omega^{m} D \delta\left(-\zeta_{1} / \zeta_{2}\right)+\frac{1}{2} \delta\left(\omega^{m} \zeta_{1} / \zeta_{2}\right) Z\left(2 m, \zeta_{2}\right) \\
-\frac{1}{2} \delta\left(\omega^{-m} \zeta_{1} / \zeta_{2}\right) Z\left(2 m, \omega^{m} \zeta_{2}\right), \quad \text { if } m=l \text { and } m+l \leq n ; \\
\frac{1}{2} \delta\left(\omega^{l} \zeta_{1} / \zeta_{2}\right) Z\left(m+l, \zeta_{2}\right)-\frac{1}{2} \delta\left(\omega^{-m} \zeta_{1} / \zeta_{2}\right) Z\left(m+l, \omega^{m} \zeta_{2}\right) \\
-\frac{1}{2} \omega^{m} \delta\left(-\omega^{l-m} \zeta_{1} / \zeta_{2}\right) Z\left(l-m, \zeta_{2}\right) \\
+\frac{1}{2} \omega^{m} \delta\left(-\zeta_{1} / \zeta_{2}\right) Z\left(l-m, \omega^{-m} \zeta_{2}\right), \\
D \delta\left(-\zeta_{1} / \zeta_{2}\right), \quad \text { if } m=l=n . \quad \text { if } m, m<l \text { and } m+l \leq n \text { and }
\end{array}\right.
\end{gathered}
$$

Now define the numbers $1=a_{0}, a_{1}, a_{2}, \ldots$ by the expansion

$$
F\left(m, l ; \omega^{-l} \zeta_{1}, \zeta_{2}\right)=\sum_{j \geq 0} a_{j}\left(\zeta_{1} / \zeta_{2}\right)^{j}
$$

where $F\left(m, l ; \omega^{-l} \zeta_{1}, \zeta_{2}\right)$ is given in (1.39). Using (1.32), (1.36), (1.41) and equating coefficients of all the monomials $\zeta_{1}^{r} \zeta_{2}^{s}(r, s \in \mathbb{Z})$ we have the following corollary of Theorem 1.1.

Corollary 1.2 [23, Theorem 2.5]. On the level one standard $\tilde{\mathfrak{g}}$-module $L(\lambda)$ (with 
$\lambda(d)=0)$, for all $r, s \in \mathbb{Z}$ and $m, l=1,2, \ldots, n$, we have

$$
\begin{array}{r}
\sum_{j \geq 0} a_{j}\left(\omega^{l j} Z(m, r-j) Z(l, s+j)-\omega^{m j} Z(l, s-j) Z(m, r+j)\right) \\
=\left\{\begin{array}{c}
-\frac{1}{2} \omega^{m} r(-1)^{r} \delta_{r+s, 0}+\frac{1}{2}\left(\omega^{m r}-\omega^{m s}\right) Z(2 m, r+s) \\
\quad \text { if } m=l \text { and } m+l \leq n \\
\frac{1}{2}\left(\omega^{l r}-\omega^{m s}\right) Z(m+l, r+s) \\
+\frac{1}{2} \omega^{m}\left(\omega^{-m s}-\omega^{l r}\right) \omega^{-m r}(-1)^{r} Z(l-m, r+s) \\
\text { if } m \neq l, m<l, \text { and } m+l \leq n \\
r(-1)^{r} \delta_{r+s, 0}, \quad \text { if } m=l=n
\end{array}\right.
\end{array}
$$

where $a_{0}, a_{1}, a_{2}, \ldots$ are defined by (1.41).

Observe that for each $r, s \in \mathbb{Z}$, the sum in (1.42) is locally finite on $L(\lambda)$ since the grading of $L(\lambda)$ is truncated from above. Hence locally, if necessary, we can compose the identities in (1.42). Now it is clear from Corollary 1.2 that for any $v \in L(\lambda) Z(m, i) \cdot v, m=2,3, \ldots, n, i \in \mathbb{Z}$, can be expressed as a linear combination of vectors of the form $Z\left(1, i_{1}\right) \cdots Z\left(1, i_{k}\right) \cdot v$, where $i_{j} \in \mathbb{Z}$. Hence it follows from (1.38) that

$$
\Omega=\operatorname{span}_{\mathbb{C}}\left\{Z\left(1, i_{1}\right) \cdots Z\left(1, i_{k}\right) \cdot v_{\lambda}\right\}
$$

where $i_{j} \in \mathbb{Z}$ for each $j$.

From here on, for convenience, we will denote the operators, $Z(1, \zeta)$, $\underline{X}(1, \zeta), X(1, \zeta), Z(1, i), \underline{X}(1, i), X(1, i)$ and $P^{ \pm}(1, \zeta)$, by $Z(\zeta), \underline{X}(\zeta)$, $X(\zeta), Z(i), \underline{X}(i), X(i)$ and $P^{ \pm}(\zeta)$ respectively.

\section{GenERATING FUNCTION IDENTITIES}

Let $\underline{L}\left(H_{i}^{*}\right)$ denote the standard $\underline{s l}(2 n, \mathbb{C})^{\sim}$-module with highest weight $H_{i}^{*}$ (where $H_{i}^{*}\left(H_{j}\right)=\delta_{i j}, H_{i}^{*}(d)=0,0 \leq i, j \leq 2 n-1$ ), $i=0,1, \ldots, n$, and highest weight vector $v_{0}$. Since $\tilde{\mathfrak{g}}=\underline{s p}(2 n, \mathbb{C})^{\sim} \subset \underline{s l}(2 n, \mathbb{C})^{\sim}$, by restriction $\underline{L}\left(H_{i}^{*}\right)$ is a $\tilde{\mathfrak{g}}$-module. Let $V$ denote the $\tilde{\mathfrak{g}}$-module generated by $v_{0}$. Note that $H_{i}^{*}\left(h_{j}\right)=\delta_{i, j}, 0 \leq i, j \leq n,($ see $(1.9)$ ). Clearly $V$ is a highest weight $\tilde{\mathfrak{g}}$-module with highest weight $h_{i}^{*}$ and highest weight vector $v_{0}$. Furthermore, since $F_{i}^{m} \cdot v_{0}=0$, for some integer $m$, and since $\left[F_{i}, F_{2 n-i}\right]=0$, for $i=1,2, \ldots, n-1$, it follows from $(1.9)$ that $f_{i}^{r} \cdot v_{0}=0, i=0,1, \ldots, n$, for some integer $r$. Hence $V$ is a standard $\tilde{\mathfrak{g}}$-module with highest weight $\lambda=h_{i}^{*}$ and highest weight vector $v_{\lambda}=v_{0}$. Since standard $\tilde{\mathfrak{g}}$-modules are unique (up to isomorphism) from here on we can and do assume that the standard $\tilde{\mathfrak{g}}$-module $L(\lambda)$ with highest weight $\lambda=h_{i}^{*}, i=0,1, \ldots, n$, is contained in the standard $\underline{s l}(2 n, \mathbb{C})^{\sim}$-module $\underline{L}\left(H_{i}^{*}\right), i=0,1, \ldots, n$, respectively.

It is known (see $[10,22])$ that for $w \in \underline{L}\left(H_{i}^{*}\right), i=0,1, \ldots, 2 n-1$,

$$
\underline{X}(m, \zeta) \cdot w=c_{i}^{(m)} \underline{E}^{-}\left(-m, \omega^{-m} \zeta\right) \underline{E}^{+}\left(-m, \omega^{-m} \zeta\right) \cdot w
$$


where

$$
c_{i}^{(m)}=\omega^{(i+1) m} /\left(\omega^{m}-1\right)
$$

for $m=1,2, \ldots, 2 n-1$. Therefore, for $v \in L\left(h_{i}^{*}\right) \subset \underline{L}\left(H_{i}^{*}\right), i=0,1, \ldots, n$, (see [23, Equation 2.16], (1.7), (1.33) and (1.34)) we have

$$
\begin{aligned}
X(\zeta) \cdot v= & X(1, \zeta) \cdot v=\frac{1}{2}[X(\zeta)+\theta X(\zeta)] \cdot v=a E^{-}\left(-1, \omega^{-m} \zeta\right) \\
\times\left[\omega^{i} \exp \left(P^{-}(\zeta)\right) \exp \left(-P^{+}(\zeta)\right)\right. & \left.\quad+\omega^{-i} \exp \left(-P^{-}(\zeta)\right) \exp \left(P^{+}(\zeta)\right)\right] E^{+}\left(-1, \omega^{-m} \zeta\right) \cdot v
\end{aligned}
$$

where $a=\frac{1}{2} \omega /(\omega-1)$. Hence it follows from (1.35) that (see [23, Equation 2.17]) we have

$$
Z(\zeta) \cdot v=a\left[\omega^{i} \exp \left(P^{-}(\zeta)\right) \exp \left(-P^{+}(\zeta)\right)+\omega^{-i} \exp \left(-P^{-}(\zeta)\right) \exp \left(P^{+}(\zeta)\right)\right] \cdot v
$$

Now looking at the homogeneous components it follows from (1.34), (1.36) and (2.4) that on $L\left(h_{i}^{*}\right), i=0,1, \ldots, n$, we have

$$
Z(j)=0, \quad \text { for all } j \text { odd } .
$$

Hence it follows from (1.43) that

$$
\Omega(L(\lambda))=\operatorname{span}_{\mathbb{C}}\left\{Z\left(2 i_{1}\right) \cdots Z\left(2 i_{k}\right) \cdot v_{\lambda} \mid k \geq 0, i_{j} \in \mathbb{Z}\right\} .
$$

Observe that for two commuting indeterminates $\zeta_{1}$ and $\zeta_{2}$ we have on $L\left(h_{i}^{*}\right)$, $i=0,1, \ldots, n,($ see $[23$, Equation 2.20])

$$
\left[P^{+}\left(\zeta_{1}\right), P^{-}\left(\zeta_{2}\right)\right]=\frac{1}{2} \log \left[\frac{\left(1-\omega^{2} \zeta_{1}^{2} / \zeta_{2}^{2}\right)\left(1-\omega^{-2} \zeta_{1}^{2} / \zeta_{2}^{2}\right)}{\left(1-\zeta_{1}^{2} / \zeta_{2}^{2}\right)^{2}}\right]
$$

where ' $\log$ ' denotes the formal logarithmic series. Hence it follows from $(2.7)$ and the Campbell-Baker-Hausdorff formula that on $L\left(h_{i}^{*}\right), i=0,1, \ldots, n$, we have

$$
\begin{aligned}
\exp \left( \pm P^{+}\left(\zeta_{1}\right)\right) \exp \left( \pm P^{-}\right. & \left.\left(\zeta_{2}\right)\right)=\exp \left( \pm P^{-}\left(\zeta_{2}\right)\right) \exp \left( \pm P^{+}\left(\zeta_{1}\right)\right) \\
& \cdot\left(1-\omega^{2} \zeta_{1}^{2} / \zeta_{2}^{2}\right)^{\frac{1}{2}}\left(1-\omega^{-2} \zeta_{1}^{2} / \zeta_{2}^{2}\right)^{\frac{1}{2}}\left(1-\zeta_{1}^{2} / \zeta_{2}^{2}\right)^{-1}
\end{aligned}
$$

and

$$
\begin{aligned}
\exp \left( \pm P^{+}\left(\zeta_{1}\right)\right) \exp \left(\mp P^{-}\left(\zeta_{2}\right)\right)=\exp \left(\mp P^{-}\left(\zeta_{2}\right)\right) \exp \left( \pm P^{+}\left(\zeta_{1}\right)\right) \\
\cdot\left(1-\omega^{2} \zeta_{1}^{2} / \zeta_{2}^{2}\right)^{-\frac{1}{2}}\left(1-\omega^{-2} \zeta_{1}^{2} / \zeta_{2}^{2}\right)^{-\frac{1}{2}}\left(1-\zeta_{1}^{2} / \zeta_{2}^{2}\right) .
\end{aligned}
$$

Now for two commuting indeterminates $\zeta_{1}$ and $\zeta_{2}$ let $F\left(\zeta_{1}, \zeta_{2}\right)$ denote the formal series $F\left(1,1 ; \zeta_{1}, \zeta_{2}\right)$, (see $(1.39)$ ) so that

$$
F\left(\zeta_{1}, \zeta_{2}\right)=\frac{\left(1-\zeta_{1} / \zeta_{2}\right)\left(1+\omega \zeta_{1} / \zeta_{2}\right)^{\frac{1}{2}}\left(1+\omega^{-1} \zeta_{1} / \zeta_{2}\right)^{\frac{1}{2}}}{\left(1+\zeta_{1} / \zeta_{2}\right)\left(1-\omega \zeta_{1} / \zeta_{2}\right)^{\frac{1}{2}}\left(1-\omega^{-1} \zeta_{1} / \zeta_{2}\right)^{\frac{1}{2}}}
$$


For a commuting set of indeterminates $\zeta_{1}, \zeta_{2}, \ldots, \zeta_{p}, p \geq 2$, we define

$$
\begin{gathered}
: Z\left(\zeta_{1}\right) \cdots Z\left(\zeta_{p}\right) \circ=\prod_{1 \leq j<k \leq p} F\left(\zeta_{j}, \zeta_{k}\right) Z\left(\zeta_{1}\right) \cdots Z\left(\zeta_{p}\right), \\
P\left(\zeta_{1}, \ldots, \zeta_{p}\right)=\prod_{1 \leq j<k \leq p}\left(1+\zeta_{j} / \zeta_{k}\right)^{2}\left(1-\omega \zeta_{j} / \zeta_{k}\right)\left(1-\omega^{-1} \zeta_{j} / \zeta_{k}\right)
\end{gathered}
$$

with $P\left(\zeta_{\sigma(1)}, \ldots, \zeta_{\sigma(p)}\right)=P\left(\zeta_{1}, \ldots, \zeta_{p}\right)$ for $\sigma \in S_{p}$ and define

$$
\begin{aligned}
& Z\left(\zeta_{1}, \ldots, \zeta_{p}\right)=P\left(\zeta_{1}, \ldots, \zeta_{p}\right)_{\circ}^{\circ} Z\left(\zeta_{1}\right) \cdots Z\left(\zeta_{p}\right)_{\circ}^{\circ} \\
& \quad=\prod_{1 \leq j<k \leq p}\left(1-\zeta_{j}^{2} / \zeta_{k}^{2}\right)\left(1-\omega^{2} \zeta_{j}^{2} / \zeta_{k}^{2}\right)^{\frac{1}{2}}\left(1-\omega^{-2} \zeta_{j}^{2} / \zeta_{k}^{2}\right)^{\frac{1}{2}} \cdot Z\left(\zeta_{1}\right) \cdots Z\left(\zeta_{p}\right) .
\end{aligned}
$$

For any two commuting indeterminates $\zeta_{1}$ and $\zeta_{2}$ we define the generalized bracket

$$
\llbracket Z\left(\zeta_{1}\right), Z\left(\zeta_{2}\right) \rrbracket=\circ Z\left(\zeta_{1}\right) Z\left(\zeta_{2}\right) \circ-\circ Z\left(\zeta_{2}\right) Z\left(\zeta_{1}\right) \circ
$$

Then it follows from Theorem 1.1 that

$$
\begin{aligned}
\llbracket Z\left(\zeta_{1}\right), Z\left(\zeta_{2}\right) \rrbracket= & -\frac{1}{2} \omega D \delta\left(-\zeta_{1} / \zeta_{2}\right)+\frac{1}{2} Z\left(2, \zeta_{2}\right) \delta\left(\omega \zeta_{1} / \zeta_{2}\right) \\
& -\frac{1}{2} Z\left(2, \omega \zeta_{2}\right) \delta\left(\omega^{-1} \zeta_{1} / \zeta_{2}\right) .
\end{aligned}
$$

Theorem 2.1. Let $\zeta_{1}, \zeta_{2}, \ldots, \zeta_{p}$ be a commuting set of indeterminates. Then for every permutation $\sigma \in S_{p}$, we have

$$
Z\left(\zeta_{\sigma(1)}, \ldots, \zeta_{\sigma(p)}\right)=Z\left(\zeta_{1}, \ldots, \zeta_{p}\right)
$$

Proof. First observe that (see [22, Lemma 2.7]) we have

$$
\begin{aligned}
& \left(1+\zeta_{1} / \zeta_{2}\right)^{2} D \delta\left(-\zeta_{1} / \zeta_{2}\right)=0, \\
& \left(1-\omega \zeta_{1} / \zeta_{2}\right) \delta\left(\omega \zeta_{1} / \zeta_{2}\right)=0, \text { and } \\
& \left(1-\omega^{-1} \zeta_{1} / \zeta_{2}\right) \delta\left(\omega^{-1} \zeta_{1} / \zeta_{2}\right)=0 .
\end{aligned}
$$

Hence for $r=1,2, \ldots, p-1$, Equation 2.15 implies

$$
\begin{aligned}
P\left(\zeta_{1}, \ldots, \zeta_{p}\right) & \prod_{\substack{1 \leq j<k \leq p \\
(j, k) \neq(r, r+1)}} F\left(\zeta_{j}, \zeta_{k}\right) \\
& \cdot Z\left(\zeta_{1}\right) \cdots Z\left(\zeta_{r-1}\right) \llbracket Z\left(\zeta_{r}\right), Z\left(\zeta_{r+1}\right) \rrbracket Z\left(\zeta_{r+2}\right) \cdots Z\left(\zeta_{p}\right)=0,
\end{aligned}
$$

and the result follows.

Let $A(\zeta)$ and $C(\xi)$ be the following Laurent series in commuting indeterminates $\zeta$ and $\xi$ with coefficients in $\operatorname{End}\left(L\left(h_{i}^{*}\right)\right), i=0,1, \ldots, n$ :

$$
A(\zeta)=\sum_{j \in \mathbb{Z}} A(j) \zeta^{j}, \quad C(\xi)=\sum_{j \in \mathbb{Z}} C(j) \xi^{j},
$$


with $[d, A(j)]=j A(j)$ and $[d, C(j)]=j C(j)$ for $j \in \mathbb{Z}$. Then

$$
A(\zeta) C(\xi)=\sum_{j, k \in \mathbb{Z}} A(j) C(k) \zeta^{j} \xi^{k}
$$

is a well-defined Laurent series in two indeterminates $\zeta$ and $\xi$, with coefficients in End $L\left(h_{i}^{*}\right)$. However, if we set $\zeta=\xi$ in $(2.18)$, the product

$$
A(\zeta) C(\zeta)=\sum_{k \in \mathbb{Z}}\left(\sum_{i+j=k} A(i) C(j)\right) \zeta^{k}
$$

is not defined in general (see [21]). Whenever the product (2.19) is defined, we write,

$$
A(\zeta) C(\zeta)=\lim _{\xi \rightarrow \zeta} A(\zeta) C(\xi)
$$

Now for products of type (2.18) involving more than two commuting indeterminates extend this definition of limit inductively (see [21]).

The next corollary follows from Theorem 2.1 and definition of limit by an argument similar to Corollary 5.8 in [21].

Corollary 2.2. For $p \geq 2$ the limit

$$
\lim _{\zeta_{1} \rightarrow \omega^{2 b_{\zeta} \zeta}} Z\left(\zeta_{1}, \zeta_{2}, \ldots, \zeta_{p}\right)
$$

exists, where $b_{1}=0, b_{2}=1, \ldots, b_{t-1}=t-2$ and for $k>t-1, b_{k}=b_{k^{\prime}}$, where $k=k^{\prime} \bmod (t-1), k^{\prime}<t-1$.

Now define $Z^{[0]}(\zeta)=1, Z^{[1]}(\zeta)=Z(\zeta)$, and for $p \geq 2$,

$$
Z^{[p]}(\zeta)=\lim _{\zeta_{l} \rightarrow \omega^{2 b_{l} \zeta}} Z\left(\zeta_{1}, \zeta_{2}, \ldots, \zeta_{p}\right)
$$

Theorem 2.3. On a level one standard $\tilde{\mathfrak{g}}$-module $L\left(h_{i}^{*}\right), i=0,1, \ldots, n$, we have

$$
Z^{[t]}(\zeta)=b Z^{[t-2]}\left(\omega^{2} \zeta\right)
$$

where ' $b$ ' is a nonzero constant independent of $i$, given $b y$

$$
b=-\frac{1}{2}(1+\omega)^{2} \prod_{1 \leq j \leq t-2}\left(1-\omega^{4 j+2}\right)\left(1-\omega^{4 j-2}\right)\left(1-\omega^{-4 j}\right)^{2} .
$$

Proof. It follows from (2.4) and (2.13) that on the $\tilde{\mathfrak{g}}$-module $L\left(h_{i}^{*}\right), \quad i=$ $0,1, \ldots, n$, we have

$$
\begin{aligned}
Z\left(\zeta_{1}, \zeta_{2}, \ldots, \zeta_{t}\right)=a^{t} \prod_{1 \leq j<k \leq t}\left(1-\zeta_{j}^{2} / \zeta_{k}^{2}\right)\left(1-\omega^{2} \zeta_{j}^{2} / \zeta_{k}^{2}\right)^{\frac{1}{2}}\left(1-\omega^{-2} \zeta_{j}^{2} / \zeta_{k}^{2}\right)^{\frac{1}{2}} \\
\quad \cdot \prod_{l=1}^{t}\left[\omega^{i} \exp \left(P^{-}\left(\zeta_{l}\right)\right) \exp \left(-P^{+}\left(\zeta_{l}\right)\right)+\omega^{-i} \exp \left(-P^{-}\left(\zeta_{l}\right)\right) \exp \left(P^{+}\left(\zeta_{l}\right)\right)\right] \\
=a^{t} \prod_{1 \leq j<k \leq t}\left(1-\zeta_{j}^{2} / \zeta_{k}^{2}\right)\left(1-\omega^{2} \zeta_{j}^{2} / \zeta_{k}^{2}\right)^{\frac{1}{2}}\left(1-\omega^{-2} \zeta_{j}^{2} / \zeta_{k}^{2}\right)^{\frac{1}{2}}
\end{aligned}
$$


(continued)

$$
\begin{aligned}
& \cdot\left[\omega^{i} \exp \left(P^{-}\left(\zeta_{1}\right)\right) \exp \left(-P^{+}\left(\zeta_{1}\right)\right)+\omega^{-i} \exp \left(-P^{-}\left(\zeta_{1}\right)\right) \exp \left(P^{+}\left(\zeta_{1}\right)\right)\right] \\
& \cdot \prod_{l=2}^{t-1}\left[\omega^{i} \exp \left(P^{-}\left(\zeta_{l}\right)\right) \exp \left(-P^{+}\left(\zeta_{l}\right)\right)+\omega^{-i} \exp \left(-P^{-}\left(\zeta_{l}\right)\right) \exp \left(P^{+}\left(\zeta_{l}\right)\right)\right] \\
& \cdot {\left[\omega^{i} \exp \left(P^{-}\left(\zeta_{t}\right)\right) \exp \left(-P^{+}\left(\zeta_{t}\right)\right)+\omega^{-i} \exp \left(-P^{-}\left(\zeta_{t}\right)\right) \exp \left(P^{+}\left(\zeta_{t}\right)\right)\right] } \\
&= a^{t} \prod_{1 \leq j<k \leq t}\left(1-\zeta_{j}^{2} / \zeta_{k}^{2}\right)\left(1-\omega^{2} \zeta_{j}^{2} / \zeta_{k}^{2}\right)^{\frac{1}{2}}\left(1-\omega^{-2} \zeta_{j}^{2} / \zeta_{k}^{2}\right)^{\frac{1}{2}} \\
& \cdot {\left[\omega^{i} \exp \left(P^{-}\left(\zeta_{1}\right)\right) \exp \left(-P^{+}\left(\zeta_{1}\right)\right)+\omega^{-i} \exp \left(-P^{-}\left(\zeta_{1}\right)\right) \exp \left(P^{+}\left(\zeta_{1}\right)\right)\right] } \\
& \cdot\left[\sum_{r=0}^{t-2} \omega^{(t-2 r-2) i} \sum_{2 \leq l_{1}<\cdots<l_{r} \leq t-1} \exp \left(P^{-}\left(\zeta_{2}\right)\right) \exp \left(-P^{+}\left(\zeta_{2}\right)\right)\right. \\
& \quad \cdots \exp \left(-P^{-}\left(\zeta_{l_{1}}\right)\right) \exp \left(P^{+}\left(\zeta_{l_{1}}\right)\right) \cdots \\
&\left.\quad \exp \left(-P^{-}\left(\zeta_{l_{r}}\right)\right) \exp \left(P^{+}\left(\zeta_{l_{r}}\right)\right) \cdots \exp \left(P^{-}\left(\zeta_{t-1}\right)\right) \exp \left(-P^{+}\left(\zeta_{t-1}\right)\right)\right] \\
&=\left[\omega^{i} \exp \left(P^{-}\left(\zeta_{t}\right)\right) \exp \left(-P^{+}\left(\zeta_{t}\right)\right)+\omega^{-i} \exp \left(-P^{-}\left(\zeta_{t}\right)\right) \exp \left(P^{+}\left(\zeta_{t}\right)\right)\right] \\
&=A_{1}+A_{2}+A_{3}+A_{4}, \operatorname{say}
\end{aligned}
$$

where

$$
\begin{aligned}
& A_{1}= a^{t} \prod_{1 \leq j<k \leq t}\left(1-\zeta_{j}^{2} / \zeta_{k}^{2}\right)\left(1-\omega^{2} \zeta_{j}^{2} / \zeta_{k}^{2}\right)^{\frac{1}{2}}\left(1-\omega^{-2} \zeta_{j}^{2} / \zeta_{k}^{2}\right)^{\frac{1}{2}} \\
& \cdot \omega^{2 i} \exp \left(P^{-}\left(\zeta_{1}\right)\right) \exp \left(-P^{+}\left(\zeta_{1}\right)\right) \\
& \cdot\left[\sum_{r=0}^{t-2} \omega^{t-2 r-2) i} \sum_{2 \leq l_{1}<\cdots<l_{r} \leq t-1} \exp \left(P^{-}\left(\zeta_{2}\right)\right) \exp \left(-P^{+}\left(\zeta_{2}\right)\right)\right. \\
& \cdots \exp \left(-P^{-}\left(\zeta_{l_{1}}\right)\right) \exp \left(P^{+}\left(\zeta_{l_{1}}\right)\right) \cdots \\
&\left.\quad \exp \left(-P^{-}\left(\zeta_{l_{r}}\right)\right) \exp \left(P^{+}\left(\zeta_{l_{r}}\right)\right) \cdots \exp \left(P^{-}\left(\zeta_{t-1}\right)\right) \exp \left(-P^{+}\left(\zeta_{t-1}\right)\right)\right] \\
& \cdot \exp \left(P^{-}\left(\zeta_{t}\right)\right) \exp \left(-P^{+}\left(\zeta_{t}\right)\right), \\
& A_{2}= a^{t} \prod_{1 \leq j<k \leq t}\left(1-\zeta_{j}^{2} / \zeta_{k}^{2}\right)\left(1-\omega^{2} \zeta_{j}^{2} / \zeta_{k}^{2}\right)^{\frac{1}{2}}\left(1-\omega^{-2} \zeta_{j}^{2} / \zeta_{k}^{2}\right)^{\frac{1}{2}} \\
& \cdot \omega^{-2 i} \exp \left(-P^{-}\left(\zeta_{1}\right)\right) \exp \left(P^{+}\left(\zeta_{1}\right)\right) \\
& \cdot\left[\sum_{r=0}^{t-2} \omega^{(t-2 r-2) i} \sum_{2 \leq l_{1}<\cdots<l_{r} \leq t-1} \exp \left(P^{-}\left(\zeta_{2}\right)\right) \exp \left(-P^{+}\left(\zeta_{2}\right)\right)\right. \\
&\left.\quad \cdots \exp \left(P^{-}\left(\zeta_{t-1}\right)\right) \exp \left(-P^{+}\left(\zeta_{t-1}\right)\right)\right]
\end{aligned}
$$


(continued)

$$
\begin{gathered}
\cdots \exp \left(-P^{-}\left(\zeta_{l_{1}}\right)\right) \exp \left(P^{+}\left(\zeta_{l_{1}}\right)\right) \\
\cdots \exp \left(-P^{-}\left(\zeta_{l_{r}}\right)\right) \exp \left(P^{+}\left(\zeta_{l_{r}}\right)\right) \\
\cdot \exp \left(-P^{-}\left(\zeta_{t}\right)\right) \exp \left(P^{+}\left(\zeta_{t}\right)\right), \\
A_{3}=a^{t} \prod_{1 \leq j<k \leq t}\left(1-\zeta_{j}^{2} / \zeta_{k}^{2}\right)\left(1-\omega^{2} \zeta_{j}^{2} / \zeta_{k}^{2}\right)^{\frac{1}{2}}\left(1-\omega^{-2} \zeta_{j}^{2} / \zeta_{k}^{2}\right)^{\frac{1}{2}} \\
\cdot \exp \left(P^{-}\left(\zeta_{1}\right)\right) \exp \left(-P^{+}\left(\zeta_{1}\right)\right) \\
\cdot\left[\sum_{r=0}^{t-2} \omega^{(t-2 r-2) i} \sum_{2 \leq l_{1}<\cdots<l_{r} \leq t-1} \exp \left(P^{-}\left(\zeta_{2}\right)\right) \exp \left(-P^{+}\left(\zeta_{2}\right)\right)\right. \\
\cdots \exp \left(-P^{-}\left(\zeta_{l_{1}}\right)\right) \exp \left(P^{+}\left(\zeta_{l_{1}}\right)\right) \\
\cdots \exp \left(-P^{-}\left(\zeta_{l_{r}}\right)\right) \exp \left(P^{+}\left(\zeta_{l_{r}}\right)\right) \\
\cdot \exp \left(-P^{-}\left(\zeta_{t}\right)\right) \exp \left(P^{+}\left(\zeta_{t}\right)\right),
\end{gathered}
$$

and

$$
\begin{aligned}
A_{4}= & a^{t} \prod_{1 \leq j<k \leq t}\left(1-\zeta_{j}^{2} / \zeta_{k}^{2}\right)\left(1-\omega^{2} \zeta_{j}^{2} / \zeta_{k}^{2}\right)^{\frac{1}{2}}\left(1-\omega^{-2} \zeta_{j}^{2} / \zeta_{k}^{2}\right)^{\frac{1}{2}} \\
& \cdot \exp \left(-P^{-}\left(\zeta_{1}\right)\right) \exp \left(P^{+}\left(\zeta_{1}\right)\right) \\
& \cdot\left[\sum_{r=0}^{t-2} \omega^{(t-2 r-2) i} \sum_{2 \leq l_{1}<\cdots<l_{r} \leq t-1} \exp \left(P^{-}\left(\zeta_{2}\right)\right) \exp \left(-P^{+}\left(\zeta_{2}\right)\right)\right. \\
& \cdots \exp \left(-P^{-}\left(\zeta_{l_{1}}\right)\right) \exp \left(P^{+}\left(\zeta_{l_{1}}\right)\right) \\
& \cdots \exp \left(-P^{-}\left(\zeta_{l_{r}}\right)\right) \exp \left(P^{+}\left(\zeta_{l_{r}}\right)\right) \\
& \cdot \exp \left(P^{-}\left(\zeta_{t}\right)\right) \exp \left(-P^{+}\left(\zeta_{t}\right)\right) .
\end{aligned}
$$

Now using equations (2.8), (2.9) and then taking limit as $\zeta_{l} \rightarrow \omega^{2 b_{l}} \zeta$, it can be easily checked that

$$
\lim _{\zeta_{l} \rightarrow \omega^{2 b_{l} \zeta}} A_{1}=\lim _{\zeta_{l} \rightarrow \omega^{2 b_{l} \zeta}} A_{2}=0
$$

and 


$$
\begin{aligned}
& \lim _{\zeta_{l} \rightarrow \omega^{2 b_{l} \zeta}} A_{3}=\lim _{\zeta_{l} \rightarrow \omega^{2 b_{l} \zeta}} A_{4}=a^{2}\left(1-\omega^{2}\right)\left(1-\omega^{-2}\right) \\
& \cdot \prod_{1 \leq j \leq t-2}\left(1-\omega^{4 j+2}\right)\left(1-\omega^{4 j-2}\right)\left(1-\omega^{-4 j}\right)^{2} \\
& \cdot \prod_{1 \leq j<k \leq t-2}\left(1-\omega^{4(j-k)}\right)\left(1-\omega^{4(j-k)+2}\right)^{\frac{1}{2}}\left(1-\omega^{4(j-k)-2}\right)^{\frac{1}{2}} \\
& \cdot a^{t-2}\left[\sum_{r=0}^{t-2} \omega^{(t-2 r-2) i} \sum_{1 \leq m_{1}<\cdots<m_{r} \leq t-2} \cdot \exp \left(P^{-}\left(\omega^{2} \zeta\right)\right) \exp \left(-P^{+}\left(\omega^{2} \zeta\right)\right)\right. \\
& \cdots \exp \left(-P^{-}\left(\omega^{2 m_{1}} \zeta\right)\right) \exp \left(P^{+}\left(\omega^{2 m_{1}} \zeta\right)\right) \\
& \cdots \exp \left(-P^{-}\left(\omega^{2 m_{r}} \zeta\right)\right) \exp \left(P^{+}\left(\omega^{2 m_{r}} \zeta\right)\right) \\
& \left.\cdots \exp \left(P^{-}\left(\omega^{2(t-2)} \zeta\right)\right) \exp \left(-P^{+}\left(\omega^{2(t-2)} \zeta\right)\right)\right] \\
& =-\frac{1}{4}(1+\omega)^{2} \prod_{1 \leq j \leq t-2}\left(1-\omega^{4 j+2}\right)\left(1-\omega^{4 j-2}\right)\left(1-\omega^{-4 j}\right)^{2} Z^{[t-2]}\left(\omega^{2} \zeta\right),
\end{aligned}
$$

since by (2.4) and (2.13) we have

$$
\begin{aligned}
& Z^{[t-2]}\left(\omega^{2} \zeta\right)=\lim _{\zeta_{l} \rightarrow \omega^{2 b_{l}+2} \zeta} Z\left(\zeta_{1}, \zeta_{2}, \ldots, \zeta_{t-2}\right) \\
& =\lim _{\zeta_{l} \rightarrow \omega^{2 l} \zeta} \prod_{1 \leq j<k \leq t-2}\left(1-\zeta_{j}^{2} / \zeta_{k}^{2}\right)\left(1-\omega^{2} \zeta_{j}^{2} / \zeta_{k}^{2}\right)^{\frac{1}{2}} \\
& \cdot\left(1-\omega^{-2} \zeta_{j}^{2} / \zeta_{k}^{2}\right)^{\frac{1}{2}} Z\left(\zeta_{1}\right) Z\left(\zeta_{2}\right) \cdots Z\left(\zeta_{t-2}\right) \\
& =a^{t-2} \lim _{\zeta_{l} \rightarrow \omega^{2 l} \zeta} \prod_{1 \leq j<k \leq t-2}\left(1-\zeta_{j}^{2} / \zeta_{k}^{2}\right)\left(1-\omega^{2} \zeta_{j}^{2} / \zeta_{k}^{2}\right)^{\frac{1}{2}}\left(1-\omega^{-2} \zeta_{j}^{2} / \zeta_{k}^{2}\right)^{\frac{1}{2}} \\
& \cdot\left[\sum_{r=0}^{t-2} \omega^{(t-2 r-2) i} \sum_{1 \leq m_{1}<\cdots<m_{r} \leq t-2} \exp \left(P^{-}\left(\zeta_{1}\right)\right) \exp \left(-P^{+}\left(\zeta_{1}\right)\right)\right. \\
& \cdots \exp \left(-P^{-}\left(\zeta_{m_{1}}\right)\right) \exp \left(P^{+}\left(\zeta_{m_{1}}\right)\right) \\
& \left.\cdots \exp \left(-P^{-}\left(\zeta_{m_{r}}\right)\right) \exp \left(P^{+}\left(\zeta_{m_{r}}\right)\right) \cdots \exp \left(P^{-}\left(\zeta_{t-2}\right)\right) \exp \left(-P^{+}\left(\zeta_{t-2}\right)\right)\right] \\
& =a^{t-2} \prod_{1 \leq j<k \leq t-2}\left(1-\omega^{4(j-k)}\right)\left(1-\omega^{4(j-k)+2}\right)^{\frac{1}{2}}\left(1-\omega^{4(j-k)-2}\right)^{\frac{1}{2}} \\
& \cdot\left[\sum_{r=0}^{t-2} \omega^{(t-2 r-2) i} \sum_{1 \leq m_{1}<\cdots<m_{r} \leq t-2} \exp \left(P^{-}\left(\omega^{2} \zeta\right)\right) \exp \left(-P^{+}\left(\omega^{2} \zeta\right)\right)\right. \\
& \cdots \exp \left(-P^{-}\left(\omega^{2 m_{1}} \zeta\right)\right) \exp \left(P^{+}\left(\omega^{2 m_{1}} \zeta\right)\right) \\
& \cdots \exp \left(-P^{-}\left(\omega^{2 m_{r}} \zeta\right)\right) \exp \left(P^{+}\left(\omega^{2 m_{r}} \zeta\right)\right) \\
& \left.\cdots \exp \left(P^{-}\left(\omega^{2(t-2)} \zeta\right)\right) \exp \left(-P^{+}\left(\omega^{2(t-2)} \zeta\right)\right)\right] .
\end{aligned}
$$


Hence we have

$$
\begin{aligned}
Z^{[t]}(\zeta) & =\lim _{\zeta_{1} \rightarrow \omega^{2 b_{l} \zeta}} Z\left(\zeta_{1}, \zeta_{2}, \ldots, \zeta_{t}\right) \\
& =\lim _{\zeta_{1} \rightarrow \omega^{2 b_{l} \zeta}}\left(A_{1}+A_{2}+A_{3}+A_{4}\right) \\
& =-\frac{1}{2}(1+\omega)^{2} \prod_{1 \leq j \leq t-2}\left(1-\omega^{4 j+2}\right)\left(1-\omega^{4 j-2}\right)\left(1-\omega^{-4 j}\right)^{2} Z^{[t-2]}\left(\omega^{2} \zeta\right)
\end{aligned}
$$

as claimed.

\section{BASES FOR LEVEL ONE STANDARD $\underline{s} p(4 k+2, \mathbb{C})^{\sim}$-MODULES}

As in $\S 2$, let $L=L(\lambda)$ denote the level one standard $\underline{s p}(2 n, \mathbb{C})^{\sim}$-module with highest weight $\lambda=h_{i}^{*}, 0 \leq i \leq n$, and highest weight vector $v_{\lambda}$ and let $\Omega(L)$ denote the corresponding vacuum space. For any sequence of integers $\mu=$ $\left(m_{1}, m_{2}, \ldots, m_{p}\right), p>0$ define the elements $Z(\mu)=Z\left(m_{1}, m_{2}, \ldots, m_{p}\right)$ in End $L(\lambda)$ by the equation

$$
Z\left(\zeta_{1}, \ldots, \zeta_{p}\right)=\sum Z\left(m_{1}, \ldots, m_{p}\right) \zeta_{1}^{m_{1}} \ldots \zeta_{p}^{m_{p}}
$$

where the summations ranges over all integers $m_{1}, \ldots, m_{p}$. Note that for $p=0$ we have the unique sequence $\mu=\varnothing$ (empty sequence) and in this case we define $Z(\varnothing)=1$. It follows immediately from equation (2.13) that

$$
Z\left(m_{1}, m_{2}, \ldots, m_{p}\right)=0
$$

unless $m_{1}, m_{2}, \ldots, m_{p} \in 2 \mathbb{Z}$ (even integers). By Theorem 2.1 we also have

$$
Z\left(m_{1}, \ldots, m_{p}\right)=Z\left(m_{\sigma(1)}, \ldots, m_{\sigma(p)}\right)
$$

for any permutation $\sigma \in S_{p}$.

For any sequence $\mu=\left(m_{1}, \ldots, m_{p}\right) \in \mathbb{Z}^{p}, p>0$, define $l(\mu)=p,|\mu|=$ $m_{1}+\cdots+m_{p}$, and write $\mu(j)=m_{j}, 1 \leq j \leq p$. Also define $l(\varnothing)=0$. For two sequences of integers $\mu=\left(m_{1}, \ldots, m_{p}\right)$ and $\nu=\left(n_{1}, \ldots, n_{p}\right), p>0$, define (cf. [25]) $\mu \geq_{T} \nu$ if and only if

$$
m_{p} \geq n_{p} ; m_{p-1}+m_{p} \geq n_{p-1}+n_{p} ; \cdots ; m_{1}+\cdots+m_{p} \geq n_{1}+\cdots+n_{p} .
$$

Then clearly for any sequence $\theta=\left(r_{1}, \ldots, r_{q}\right), q \geq 0$, of integers we have

$$
\mu \geq_{T} \nu \Rightarrow \mu \circ \theta \geq_{T} \nu \circ \theta \text { and } \theta \circ \mu \geq_{T} \theta \circ \nu,
$$

where the composition is defined by juxtaposition, i.e.

$$
\mu \circ \theta=\left(m_{1}, \ldots, m_{p}, r_{1}, \ldots, r_{q}\right) \text {. }
$$

Lemma 3.1. Let us define the coefficients $a(\mu)$ and $b(\mu)$ by the formal identities:

$$
\prod_{1 \leq j<k \leq p}\left(1-\zeta_{j}^{2} / \zeta_{k}^{2}\right)\left(1-\omega^{2} \zeta_{j}^{2} / \zeta_{k}^{2}\right)^{\frac{1}{2}}\left(1-\omega^{-2} \zeta_{j}^{2} / \zeta_{k}^{2}\right)^{\frac{1}{2}}=\sum_{\mu \in \mathbb{Z}^{p}} a(\mu) \zeta_{1}^{\mu(1)} \cdots \zeta_{p}^{\mu(p)},
$$


and

$\prod_{1 \leq j<k \leq p}\left(1-\zeta_{j}^{2} / \zeta_{k}^{2}\right)^{-1}\left(1-\omega^{2} \zeta_{j}^{2} / \zeta_{k}^{2}\right)^{-\frac{1}{2}}\left(1-\omega^{-2} \zeta_{j}^{2} / \zeta_{k}^{2}\right)^{-\frac{1}{2}}=\sum_{\mu \in \mathbb{Z}^{p}} b(\mu) \zeta_{1}^{\mu(1)} \cdots \zeta_{p}^{\mu(p)}$.

Then $a(\mathbf{O})=1=b(\mathbf{O})$ and $a(\mu)=0=b(\mu)$ unless $\mu \leq_{T} \mathbf{O}$, where $\mathbf{O}=$ $(0, \ldots, 0)$. Furthermore,

$$
Z\left(m_{1}, \ldots, m_{p}\right)=\sum_{\mu \in \mathbb{Z}^{p}} a(\mu) Z\left(m_{1}-\mu(1)\right) \cdots Z\left(m_{p}-\mu(p)\right),
$$

and

$$
Z\left(m_{1}\right) \cdots Z\left(m_{p}\right)=\sum_{\mu \in \mathbb{Z}^{p}} b(\mu) Z\left(m_{1}-\mu(1), \ldots, m_{p}-\mu(p)\right) .
$$

Proof. For (1) compare the coefficient of $\zeta_{1}^{m_{1}} \zeta_{2}^{m_{2}} \ldots \zeta_{p}^{m_{p}}$ in equation (3.1). For (2) multiply equation (3.1) by

$$
\prod_{1 \leq j<k \leq p}\left(1-\zeta_{j}^{2} / \zeta_{k}^{2}\right)^{-1}\left(1-\omega^{2} \zeta_{j}^{2} / \zeta_{k}^{2}\right)^{-\frac{1}{2}}\left(1-\omega^{-2} \zeta_{j}^{2} / \zeta_{k}^{2}\right)^{-\frac{1}{2}}
$$

and then compare the coefficients of $\zeta_{1}^{m_{1}} \zeta_{2}^{m_{2}} \ldots \zeta_{p}^{m_{p}}$.

The next corollary follows immediately from Lemma 3.1.

Corollary 3.2. For $\mu=\left(m_{1}, m_{2}, \ldots, m_{p}\right) \in \mathbb{Z}^{p}$, we have

$$
\begin{aligned}
& Z(\mu)=Z\left(m_{1}\right) \cdots Z\left(m_{p}\right)+\sum_{\nu>T_{T} \mu} a(\mu-\nu) Z(\nu(1)) \cdots Z(\nu(p)), \\
& Z\left(m_{1}\right) \cdots Z\left(m_{p}\right)=Z(\mu)+\sum_{\nu>_{T} \mu} b(\mu-\nu) Z(\nu) .
\end{aligned}
$$

Corollary 3.3. We have

$$
\begin{aligned}
& Z\left(m_{1}, \ldots, m_{p}\right) Z\left(n_{1}, \ldots, n_{r}\right) \\
& \quad=Z\left(m_{1}, \ldots, m_{p}, n_{1}, \ldots, n_{r}\right)+\sum_{\nu>>_{T}\left(m_{1}, \ldots, m_{p}, n_{1}, \ldots, n_{r}\right)} c(\nu) Z(\nu)
\end{aligned}
$$

for some scalars $c(\nu)$.

Proof. It is clear from Corollary $3.2(1)$ and (3.4) that

$$
\begin{gathered}
Z\left(m_{1}, \ldots, m_{p}\right) Z\left(n_{1}, \ldots, n_{r}\right)=Z\left(m_{1}\right) \cdots Z\left(m_{p}\right) Z\left(n_{1}\right) \cdots Z\left(n_{r}\right) \\
\quad+\sum_{\nu>{ }_{T}\left(m_{1}, \ldots, m_{p}, n_{1}, \ldots, n_{r}\right)} d(\nu) Z(\nu(1)) \cdots Z(\nu(p+r))
\end{gathered}
$$

for some scalars $d(\nu)$. Now the result follows from Corollary $3.2(2)$.

For any two sequences $\mu$ and $\nu$, we say $\mu<\nu$ if and only if $l(\mu)>l(\nu)$ or $\mu<_{T} \nu$. Then it follows from (3.4) that

$$
\mu<\nu \Rightarrow \mu \circ \theta<\nu \circ \theta \text { and } \theta \circ \mu<\theta \circ \nu
$$


for any sequence $\theta$. Let $\mathscr{P}$ denote the set of all sequences $\mu=\left(2 m_{1}, \ldots, 2 m_{p}\right)$ $\in(2 \mathbb{Z})^{p}, p \geq 0$, such that $m_{1} \leq m_{2} \leq \cdots \leq m_{p}<0$. For any standard $\tilde{\mathfrak{g}}^{-}$ module $L=L(\lambda)$ with highest weight $v_{\lambda}$, where $\lambda=h_{i}^{*}, 0 \leq i \leq n$, define

$$
\Omega(L)_{(\mu)}=\sum_{\substack{\nu>\mu \\ \nu \in \mathscr{P}}} \mathbb{C} Z(\nu) v_{\lambda},
$$

for all $\mu \in \mathscr{P}, \mu \neq \varnothing$ and define $\Omega(L)_{(\varnothing)}=\{0\}$. Clearly, we have

$$
\Omega(L)_{(\mu)} \supseteq \Omega(L)_{(\nu)}, \quad \text { for } \mu \leq \nu ; \mu, \nu \in \mathscr{P} .
$$

Proposition 3.4. $\Omega=\Omega(L)=\bigcup_{\mu \in \mathscr{P}} \Omega(L)_{(\mu)}$.

Proof. It follows from (2.6), (3.3) and Corollary 3.2 that

$$
\Omega=\operatorname{span}_{\mathbb{C}}\left\{Z(\mu) v_{\lambda} \mid \mu=\left(m_{1}, \ldots, m_{p}\right) \in(2 \mathbb{Z})^{p}, p \geq 0, m_{1} \leq \cdots \leq m_{p}\right\} .
$$

Let

$$
V=\operatorname{span}_{\mathbb{C}}\left\{Z(\nu) v_{\lambda} \mid \nu \in \mathscr{P}\right\} .
$$

Clearly $V \subseteq \Omega$. To show $\Omega \subseteq V$ we use induction on the ordering of the sequences $\mu=\left(m_{1}, \ldots, m_{p}\right) \in(2 \mathbb{Z})^{p}, p \geq 0$. Fix $\mu=\left(m_{1}, \ldots, m_{p}\right) \in$ $(2 \mathbb{Z})^{p}, m_{1} \leq \cdots \leq m_{p}$. Assume that $Z\left(\mu^{\prime}\right) v_{\lambda} \in V$ for all $\mu^{\prime}>\mu$. We want to show that $Z(\mu) v_{\lambda} \in V$. Suppose $\mu=\left(m_{1}, \ldots, m_{r}, m_{r+1}, \ldots, m_{p}\right)$, where $m_{1} \leq \cdots \leq m_{r}<0$ and $0 \leq m_{r+1} \leq \cdots \leq m_{p}$. By Corollary 3.3 we have

$$
Z(\mu) v_{\lambda}=Z\left(m_{1}, \ldots, m_{r}\right) Z\left(m_{r+1}, \ldots, m_{p}\right) v_{\lambda}+\sum_{\mu^{\prime}>\mu} c\left(\mu^{\prime}\right) Z\left(\mu^{\prime}\right) v_{\lambda} .
$$

Hence by assumption, it is enough to show that

$$
Z\left(m_{1}, \ldots, m_{r}\right) Z\left(m_{r+1}, \ldots, m_{p}\right) v_{\lambda} \in V .
$$

If $r>0$, then this follows from the induction hypothesis and Corollary 3.3 since $\left(m_{r+1}, \ldots, m_{p}\right)>\mu$. Now suppose $r=0$. Then we have $0 \leq m_{1} \leq \cdots \leq m_{p}$ and by Corollary 3.2,

$$
Z(\mu) v_{\lambda}=Z\left(m_{1}\right) \cdots Z\left(m_{p}\right) v_{\lambda}+\sum_{\mu^{\prime}>\mu} d\left(\mu^{\prime}\right) Z\left(\mu^{\prime}\right) v_{\lambda},
$$

where $d\left(\mu^{\prime}\right)$ are some scalars. But since $v_{\lambda}$ is a highest weight vector and $0 \leq m_{1} \leq \cdots \leq m_{p}$, so $Z\left(m_{1}\right) \cdots Z\left(m_{p}\right) v_{\lambda}$ is a scalar multiple of $v_{\lambda}$. Hence it again follows by the induction hypothesis that $Z(\mu) v_{\lambda} \in V$.

For $p \geq 1$ and $r \in \mathbb{Z}$ denote by $(p ; r)$ the unique sequence of integers (see [21]),

such that

$$
(p ; r)=\left(m_{1}, \ldots, m_{p}\right)
$$

$$
\begin{gathered}
r=m_{1}+\cdots+m_{p}, \quad m_{1} \leq \cdots \leq m_{p} \quad \text { and } \\
0 \leq m_{p}-m_{1} \leq 1
\end{gathered}
$$

Let $2(p ; r)$ denote the corresponding unique sequence of even integers $\left(2 m_{1}\right.$, $\left.\ldots, 2 m_{p}\right)$. The following lemma is clear (see [21, Lemma 8.2]). 
Lemma 3.5. Let $\mu=\left(2 n_{1}, \ldots, 2 n_{p}\right) \in \mathscr{P}, p>0$, and $|\mu|=2 r$. Then we have

$$
\begin{gathered}
2(p ; r) \leq \mu, \\
\text { if } \mu \neq 2(p, r), \text { then } n_{1} \leq-2+n_{p} .
\end{gathered}
$$

For $p \geq 2$, let

$$
Z^{[p]}(\zeta)=\sum_{r \in \mathbb{Z}} Z^{[p]}(r) \zeta^{r}
$$

It follows from (2.5), (2.13) and (2.21) that $Z^{[p]}(r)=0$ unless $r \in 2 \mathbb{Z}$. The next proposition follows immediately from (2.13), (2.21) and Lemma 3.5.

Proposition 3.6. For $r \in \mathbb{Z}$ and $p \geq 2$, we have

$$
Z^{[p]}(2 r)=e Z(2(p ; r))+\sum_{\nu>2(p ; r)} e_{\nu} Z(\nu)
$$

for some nonzero scalar $e$ and scalars $e_{\nu}$ 's and $\nu \in \mathscr{P}$.

For a sequence $\mu=\left(2 m_{1}, \ldots, 2 m_{p}\right) \in(2 \mathbb{Z})^{p}, m_{1} \leq \cdots \leq m_{p}$, we say that $\mu$ satisfies the difference two condition if for every $r \in\{1, \ldots, p-t+1\}$ we have $m_{r} \leq-2+m_{r+t-1}$. Observe that for $s \in \mathbb{Z}$ the sequence $2(t ; s)$ does not satisfy the difference two condition.

Theorem 3.7. If $\mu \in \mathscr{P}$ does not satisfy the difference two condition, then $Z(\mu) v_{\lambda}$ $\in \Omega(L)_{\mu}$ for $\lambda=h_{i}^{*}, i=0,1, \ldots, n$.

Proof. Since $\mu \in \mathscr{P}$ does not satisfy the difference two condition, so it must be of the form

$$
\mu=\left(2 m_{1}, \ldots, 2 m_{s}, 2(t ; r), 2 m_{s+t+1}, \ldots, 2 m_{p}\right) .
$$

Let

$$
2(t ; r)=\left(2 m_{s+1}, \ldots, 2 m_{s+t}\right) .
$$

Then Theorem 2.3 along with equation (3.8) implies that for any $v \in L=$ $L\left(h_{i}^{*}\right), i=0,1, \ldots, n$,

$$
Z^{[t]}(2 r) v=\sum_{l(\nu)<t} b_{\nu} Z(\nu) v,
$$

for some scalars $b_{\nu}$. This together with Proposition 3.6 then implies that

$$
Z(2(t ; r)) v=\sum_{\nu>2(t ; r)} a_{\nu} Z(\nu) v,
$$

for some scalars $a_{\nu}$. Now set $v=Z\left(2 m_{s+t+1}, \ldots, 2 m_{p}\right) v_{\lambda}$ and multiply (3.9) from the left by $Z\left(2 m_{1}, \ldots, 2 m_{s}\right)$. Now thanks to Corollary 3.3 and equations (3.2), (3.3), (3.5) we have the desired result.

It is clear from (2.4) that

$$
Z(0) v_{\lambda}=a\left(\omega^{i}+\omega^{-i}\right) v_{\lambda}
$$


for $\lambda=h_{i}^{*}, 0 \leq i \leq n$, where $a=\frac{1}{2} \omega /(\omega-1)$. For $r \geq 0$, write

$$
\mu(r)=(-2, \ldots,-2) \in(2 \mathbb{Z})^{r} .
$$

For $p \geq r$, write

$$
\mu_{p}(r)=(-2, \ldots,-2,0, \ldots, 0) \in(2 \mathbb{Z})^{p}
$$

with $r$ entries equal to -2 . The next lemma follows from (3.10) and Corollary 3.2 .

Lemma 3.8. For $p \geq r, \lambda=h_{i}^{*}, i=0,1, \ldots, n$, we have

$$
Z\left(\mu_{p}(r)\right) v_{\lambda}=\left(a\left(\omega^{i}+\omega^{-i}\right)\right)^{p-r} Z(\mu(r)) v_{\lambda}+\sum_{\nu>\mu(r)} b_{\nu} Z(\nu) v_{\lambda},
$$

for some scalars $b_{\nu}$.

Remark 3.9. For $\lambda=h_{n-i}^{*}$, we have from equation (3.10),

$$
Z(0) v_{\lambda}=a\left(\omega^{n-i}+\omega^{-(n-i)}\right) v_{\lambda}=-a\left(\omega^{i}+\omega^{-i}\right) v_{\lambda}
$$

since $\omega^{n}=-1=\omega^{-n}$.

Lemma 3.10. For $p \geq r, \lambda=h_{i}^{*}$ or $h_{n-i}^{*}, i=0,1, \ldots, t-1$, there is $a$ polynomial $f_{t, p, r}(x)$ of degree $p-r$ which is independent of $i$ such that the coefficient of $\zeta_{1}^{-2} \zeta_{2}^{-2} \ldots \zeta_{r}^{-2}$ in $Z\left(\zeta_{1}, \ldots, \zeta_{p}\right) v_{\lambda}$ is of the form

$$
f_{t, p, r}\left( \pm a\left(\omega^{i}+\omega^{-i}\right)\right) Z(\mu(r)) v_{\lambda}+\sum_{\nu>\mu(r)} d_{\nu} Z(\nu) v_{\lambda}
$$

for some scalars $d_{\nu}$. Here the sign is + or - depending on $\lambda=h_{i}^{*}$ or $\lambda=h_{n-i}^{*}$, $i=0,1, \ldots, t-1$.

Proof. It follows from Theorem 2.1 and equations (3.1), (3.2) that

$$
Z\left(\zeta_{1}, \ldots, \zeta_{p}\right)=\sum_{\sigma \in S_{p}}\left(\sum_{m_{1} \leq \cdots \leq m_{p}} Z\left(2 m_{1}, \ldots, 2 m_{p}\right)\right) \zeta_{1}^{2 m_{\sigma(1)}} \ldots \zeta_{p}^{2 m_{\sigma(p)}} .
$$

Hence the coefficient of $\zeta_{1}^{-2} \ldots \zeta_{r}^{-2}$ in $Z\left(\zeta_{1}, \ldots, \zeta_{p}\right) v_{\lambda}$ is a linear combination of terms of the form $Z(\mu) v_{\lambda}$ where $\mu=\left(2 m_{1}, \ldots, 2 m_{s}\right), s \leq p, m_{1} \leq \cdots \leq$ $m_{s}$ and $m_{1}+\cdots+m_{s}=-r$. Since the coefficient of $Z(\mu(r)) v_{\lambda}$ is nonzero and also if $\mu>_{T} \mu_{s}(r)$ then $Z(\mu) v_{\lambda} \in \Omega(L)_{(\mu(r))}$, therefore the result follows from Lemma 3.8, Corollary 3.2 and Remark 3.9.

Proposition 3.11. For $r \leq t, \lambda=h_{i}^{*}$ or $h_{n-i}^{*}, i=0,1, \ldots, t-1$, there is $a$ polynomial $g_{t, r}(x)$ of degree $t-r$, independent of $i$, such that

$$
g_{t, r}\left( \pm a\left(\omega^{i}+\omega^{-i}\right)\right) Z(\mu(r)) v_{\lambda} \in \Omega(L)_{(\mu(r))} .
$$

Here the sign is + or - depending on $\lambda=h_{i}^{*}$ or $h_{n-i}^{*}, 0 \leq i \leq t-1$.

Proof. From Theorem 2.3, we have

$$
\lim _{\zeta_{1} \rightarrow \omega^{2 b_{\zeta} \zeta}}\left[Z\left(\zeta_{1}, \ldots, \zeta_{t}\right)-b Z\left(\zeta_{1}, \ldots, \zeta_{t-2}\right)\right] v_{\lambda}=0
$$


where ' $b$ ' is a nonzero scalar independent of $i$. In (3.13), first collecting the coefficients of $\zeta_{1}^{-2} \cdots \zeta_{r}^{-2}$ by using Lemma 3.10 and then taking the limit we get

$$
\left[f_{t, t, r}\left( \pm a\left(\omega^{i}+\omega^{-i}\right)\right)-b f_{t, t-2, r}\left( \pm a\left(\omega^{i}+\omega^{-i}\right)\right)\right] \cdot Z(\mu(r)) v_{\lambda} \in \Omega(L)_{\mu(r)}
$$

Now setting $g_{t, r}(x)=f_{t, t, r}(x)-b f_{t, t-2, r}(x)$, the result follows from (3.14).

The following lemma is an immediate consequence of the character formulas (1.26) and (1.27).

Lemma 3.12. If $r \leq i, \lambda=h_{i}^{*}$ or $h_{n-i}^{*}, i=0,1, \ldots, t-1$, then $Z(\mu(r)) v_{\lambda} \notin$ $\Omega(L)_{(\mu(r))}$.

Proposition 3.13. For $\lambda=h_{i}^{*}$ or $h_{n-i}^{*}, i=0,1, \ldots, t-1$, we have $Z(\mu(i+1)) v_{\lambda} \in \Omega(L)_{(\mu(i+1))}$.

Proof. First let us consider the case when $\lambda=h_{i}^{*}, i=0,1, \ldots, t-1$. If $r \leq t$, then by Proposition 3.11 and Lemma 3.12, we have

$$
g_{t, r}\left(a\left(\omega^{i}+\omega^{-i}\right)\right)=0 \text { for } i=r, r+1, \ldots, t-1 .
$$

But since $g_{t, r}$ has degree $t-r$, this implies that for $i<r$

$$
g_{t, r}\left(a\left(\omega^{i}+\omega^{-i}\right)\right) \neq 0 \text {. }
$$

Now setting $r=i+1$ in (3.15), we have $g_{t, i+1}\left(a\left(\omega^{i}+\omega^{-i}\right)\right) \neq 0$. Hence by Proposition 3.11, we have $Z(\mu(i+1)) v_{\lambda} \in \Omega(L)_{\mu(i+1)}$ as desired. The case $\lambda=h_{n-i}^{*}, 0 \leq i \leq t-1$ follows similarly by replacing ' $a$ ' with ' $-a$ ' in the above argument.

We say that a sequence $\mu=\left(2 m_{1}, \ldots, 2 m_{p}\right) \in \mathscr{P}$ satisfies the initial condition if at most $i(0 \leq i \leq t-1)$ elements $m_{s}$ are equal to -1 .

Theorem 3.14. For $\lambda=h_{i}^{*}$ or $h_{n-i}^{*}, 0 \leq i \leq t-1$, if $\mu \in \mathscr{P}$ does not satisfy the initial condition, then $Z(\mu) v_{\lambda} \in \Omega(L)_{(\mu)}$.

Proof. Since $\mu \in \mathscr{P}$ does not satisfy the initial condition, so it must be of the form

where

$$
\mu=\left(2 m_{1}, \ldots, 2 m_{p-i-1}, 2 m_{p-i}, \ldots, 2 m_{p}\right)
$$

$$
m_{p-i}=\cdots=m_{p}=-1 \text {. }
$$

Set $\mu^{\prime}=\left(2 m_{1}, \ldots, 2 m_{p-i-1}\right)$. Then $\mu=\mu^{\prime} \circ \mu(i+1)$. By Corollary 3.3, we have

$$
Z(\mu) v_{\lambda}=Z\left(\mu^{\prime}\right) Z(\mu(i+1)) v_{\lambda}+\sum_{\nu>\mu} e_{\nu} Z(\nu) v_{\lambda},
$$

for some scalars $e_{\nu}$. Now the desired result follows thanks to Proposition 3.13, Corollary 3.3 and equation (3.5).

For $\lambda=h_{i}^{*}$ or $h_{n-i}^{*}, 0 \leq i \leq t-1$, denote by $\mathscr{C}_{\lambda}$ the set of all $\mu \in \mathscr{P}$ such that $\mu$ satisfies the difference two condition and the initial condition. 
Theorem 3.15. For $\lambda=h_{i}^{*}$ or $h_{n-i}^{*}, 0 \leq i \leq t-1$, let $L(\lambda)$ denote the standard $\tilde{\mathfrak{g}}$-module with highest weight $\lambda$ and highest weight vector $v_{\lambda}$. Let $\Omega(L)$ denote the vacuum space of $L(\lambda)$. The set of vectors $\left\{Z(\mu) v_{\lambda} \mid \mu \in \mathscr{C}_{\lambda}\right\}$ spans $\Omega(L)$.

Proof. By Proposition 3.4, we have that the set of vectors $\left\{Z(\mu) v_{\lambda} \mid \mu \in \mathscr{P}\right\}$ span the space $\Omega(L)$. Now using Theorems 3.7 and 3.14, the desired result follows by induction on the ordering of $\mathscr{P}$.

Observe that for $\mu \in \mathscr{C}_{\lambda}$, the vector $Z(\mu) v_{\lambda} \in \Omega(L)_{|\mu|}$. Hence in the case when $n=2 k+1$, the following theorem is an immediate consequence of Theorem 3.15 and the character formula (1.26) of $\Omega(L)$ together with the generalized Rogers-Ramanujan identities (see [1, 2]) due to Gordon, Andrews and Bressoud.

Theorem 3.16. For $\lambda=h_{i}^{*}$ or $h_{n-i}^{*}, 0 \leq i \leq k$, let $L(\lambda)$ denote the standard $\underline{s p}(4 k+2, \mathbb{C})^{\sim}$-module with highest weight $\lambda$ and highest weight vector $v_{\lambda}$. Let $\bar{\Omega}(L)$ denote the vacuum space of $L(\lambda)$. The set of vectors $\left\{Z(\mu) v_{\lambda} \mid \mu \in \mathscr{C}_{\lambda}\right\}$ is a basis of $\Omega(L)$.

\section{REFERENCES}

1. G. E. Andrews, The theory of partitions, Encyclopedia of Mathematics and its Applications, Vol. 2, G.-C. Rota (ed.), Addison-Wesley, Reading, Mass., 1976.

2. D. Bressoud, Analytic and combinatorial generalizations of the Rogers-Ramanujan identities, Mem. Amer. Math. Soc. 24 (1980), no. 227.

3. D. Bernard and J. Thierry-Mieg, Level one representations of the simple affine Kac-Moody algebras in their homogeneous gradations, Comm. Math. Phys. 111 (1987), 181-246.

4. I. B. Frenkel, Two constructions of affine Lie algebra representations and Boson-Fermion correspondence in quantum field theory, J. Funct. Anal. 44 (1981), 259-327.

5. I. B. Frenkel and V. G. Kac, Basic representations of affine Lie algebras and dual resonance models, Invent. Math. 62 (1980), 23-66.

6. P. Goddard, W. Nahm, D. Olive and A. Schwimmer, Vertex operators for non-simply laced algebras, Comm. Math. Phys. 107 (1986), 179-212.

7. V. G. Kac, Infinite-dimensional Lie algebras (2nd ed.), Cambridge Univ. Press, Cambridge, 1985.

8. V. G. Kac and D. H. Peterson, Spin and wedge representations of infinite-dimensional Lie algebras and groups, Proc. Nat. Acad. Sci. U.S.A. 78 (1981), 3308-3312.

9. __ 112 constructions of the basis representation of the loop group of $E_{8}$, Symposium on Anomalies, Geometry, Topology, 1985, Proceedings, W. A. Bardeen and A. R. White (eds.), World Scientific, Singapore, 1985, pp. 276-298.

10. V. G. Kac, D. A. Kazhdan, J. Lepowsky and R. L. Wilson, Realization of basic representations of the euclidean Lie algebras, Adv. in Math. 42 (1981), 83-112.

11. J. Lepowsky, Lectures on Kac-Moody Lie algebras, Université Paris VI, Spring, 1978.

12. __, Calculus of twisted vertex operators, Proc. Nat. Acad. Sci. U.S.A. 82 (1985), 82958299.

13. J. Lepowsky and M. Primc, Standard modules for type one affine Lie algebras, Number Theory, Lecture Notes in Math., vol. 1052, Springer-Verlag, New York, 1984, pp. 194-251.

14. _ Structure of the standard modules for the affine Lie algebra $A_{1}^{(1)}$, Contemp. Math., vol. 46, Amer. Math. Soc., Providence, R.I., 1985. 
15. J. Lepowsky and R. L. Wilson, Construction of the affine Lie algebra $A_{1}^{(1)}$, Comm. Math. Phys. 62 (1978), 43-53.

16. __ A new family of algebras underlying the Rogers-Ramanujan identities and generalizations, Proc. Nat. Acad. Sci. U.S.A. 78 (1981), 7254-7258.

17. _ A Lie theoretic interpretation and proof of the Rogers-Ramanujan identities, Adv. in Math. 45 (1982), 21-72.

18. - The structure of standard modules, I. Universal algebras and the Rogers-Ramanujan identities, Invent. Math. 77 (1984), 199-290.

19. __ The structure of standard modules, II. The case $A_{1}^{(1)}$, principal gradation, Invent. Math. 79 (1985), 417-442.

20. M. Mandia, Structure of the level one standard modules for the affine Lie algebras $B_{l}^{(1)}, F_{4}^{(1)}$ and $G_{2}^{(1)}$, Mem. Amer. Math. Soc. 362 (1987).

21. A. Meurman and M. Primc, Annihilating ideals of standard modules of $\underline{s l}(2, \mathbb{C})^{\sim}$ and combinatorial identities, Adv. in Math. 64 (1987), 177-240.

22. K. C. Misra, Structure of certain standard modules for $A_{n}^{(1)}$ and the Rogers-Ramanujan identities, J. Algebra 88 (1984), 196-227.

23. __ Structure of some standard modules for $C_{n}^{(1)}$, J. Algebra 90 (1984), 384-409.

24. ___ Constructions of fundamental representations of symplectic affine Lie algebras, Topological and Geometrical Methods in Field Theory, J. Hietarinta and J. Westerholm (eds.), World Scientific, Singapore, 1986, pp. 147-169.

25. __ Realization of the level two standard $\underline{s}(2 k+1, \mathbb{C})^{\sim}$-modules, Trans. Amer. Math. Soc. 316 (1989), 295-309.

26. G. Segal, Unitary representations of some infinite-dimensional groups Comm. Math. Phys. 80 (1981), 301-342.

Department of Mathematics, North Carolina State University, Raleigh, North CarOLINA 27695-8205 